\title{
Immediate Effect of Buteyko Breathing Exercise and Coherent Breathing Exercise on Subjects with Primary Dysmenorrhoea: A Randomised Clinical Trial
}

\author{
Bulbuli. A, Joshi.I, Jadhav. A and Porwal. J
}

\begin{abstract}
Aim: To compare the effect of Buteyko breathing exercise and Coherent breathing exercise in subjects with primary dysmenorrhoea. Method: Fifteen $(\mathrm{N}=15)$ subjects participated in the present study which were further divided into two groups and their age ranged from 18 to 24 years. The outcome measure used were Numeric pain rating scale and Moos menstrual distress questionnaire. The pre and post intervention measurements were obtained and analyzed. Results: Statistically both Group A (Buteyko breathing exercise) and Group B (Coherent breathing exercise) showed significant 5\% reduction in pain intensity but Coherent breathing exercise showed better results than Buteyko breathing exercise. Conclusion: The result of the present study showed that Coherent breathing exercise was better than Buteyko breathing exercise in subject's with primary dysmenorrhoea.
\end{abstract}

Bulbuli. A

Assistant Professor

Kaher Institute of Physiotherapy

Belgavi 590010 Karnataka

Joshi.I

BPT Intern

Kaher Institute of Physiotherapy

Belgavi 590010 Karnataka

E-mail: ishajoshi48@gmail.com

Jadhav.A

BPT Intern

Kaher Institute of Physiotherapy

Belgavi 590010 Karnataka

Porwal.J

BPT Intern

Kaher Institute of Physiotherapy

Belgavi 590010 Karnataka
Key Words: NPRS, MMDQ, Menstrual Pain

DOI: 10.18376/jesp/2019/v15/i1/111322

\section{Introduction}

Menstruation is the noticeable uterine bleeding due to periodic physiological shedding of the endometrium caused due to interaction of hormones. The time span between two consecutive menstruations is termed as one menstrual cycle. Uterine bleeding occurs for about 4-5days with an average blood loss of $35 \mathrm{ml}$, out of which $70 \%$ of blood loss occurs in first two days of menstruation (Dutta 2008). Dysmenorrhoea is painful menstrual bleeding of high intensity which hampers daily routine of an individual (Dutta 2008). It is one of the most common gynaecological condition which causes absenteeism from work and school during the menstrual event (Proctor and Farquhar 2006).Menstrual cramps mean perception of pain during menstruation whereas dysmenorrhoea means high intensity pain which renders individual incapable of 


\section{Journal of Exercise Science \& Physiotherapy Vol. 15 No. 1 (January to June) 2019 \\ ISSN: 0973-2020 (Print) $\quad \mathrm{I}_{2} \mathrm{OR}$ Impact Factor $=6.502 \quad$ ISSN: 2454-6089 (Online)}

functioning normally in their daily activities and ultimately calls for the need for medications (Grandi et. al., 2012).Menstrual pain without any known pelvic pathology is termed as primary dysmenorrhoea. Many studies conducted showed a positive correlation between primary dysmenorrhoea and duration of menstrual flow, younger age of menarche, increased BMI and cigarette smoking (Sundell et. al.,1990 and Chen et. al.,2000).One of the predominant and most common causes of primary dysmenorrhoea is increased production of prostaglandins from the endometrium or increased sensitivity of myometrium to prostaglandins (Iacovides et. al., 2014b). Primary dysmenorrhoea is prevalent in about 70-90 percent of teenage girls and 30-50 percent of menstruating women in India (Kaur et. al., 2017). In the ovulatory phase of the menstrual cycle, there is synthesis of prostaglandins under the influence of the hormone progesterone. The synthesis reaches its peak during the shedding of myometrium. Prostaglandins being a vasoconstrictor, it causes ischemia of myometrium which causes pain perception during periods. Prostaglandin $\mathrm{PGF}_{2} \alpha$ is also responsible for increased myometrial contraction which adds up to the increased perception of pain during periods (Dutta 2008). Physical symptoms include Abdominal bloating, abdominal pain radiating to thigh and lower back, breast heaviness are present during the periods, these can also be present before the start of menstrual bleeding and is termed as pre menstrual syndrome(PMS). It is found that girls with premenstrual syndrome had 56.1 per cent chances of having dysmenorrhoea than the girls without any pre menstrual symptoms. Breathing exercise when combined with relaxation has positive effect on pain perception. Buteyko breathing exercise and coherent breathing technique helps in reduction of pain by their said mechanisms. The main principle of Buteyko exercise is normalization of breathing pattern. It consists of three components namely- nasal breathing, control pause, and relaxation (Pal and Velkumary 2004). Coherent breathing focuses on breathing at a frequency of 5 breaths per minute. Its main objective is to stimulate the parasympathetic nervous system and hence cause relaxation (Markum 1976).

\section{Materials and Method}

The present study was conducted on fifteen $(\mathrm{N}=1)$ subjects which were divided into two groups within the age ranged from 18 to 24 years. The outcome measure used were Numeric pain rating scale and MOOS menstrual distress questionnaire. Demographic data like age height, weight and BMI were taken before the intervention. The pre and post intervention measurements were obtained and analyzed.

Inclusion/ Exclusion Criteria: Nulliparous females with primary dysmenorrhoea between the age group of 18-24 years were included in the study. Subjects with secondary dysmenorrhoea, Subjects who are on dysmenorrhoea medications, subjects with respiratory, cardiac or neurological conditions or with recent abdominal or cardiothoracic surgery were excluded from the study.

Outcome Measures: Two outcome measures were taken MOOS menstrual distress questionnaire is a questionnaire containing 47 symptoms on a 6 point scale. The 47 symptoms are inter correlated clusters of symptoms like labelled pain, concentration, behavioural change, autonomic reactions, water retention, negative effect, arousal and control as the MMDQ takes only about 5 minutes or woman to fill and can be given repeatedly, it is a method that implies patients own subjective assessment of the extent disability and hence provides an essential source of information (Choudhury et. al., 2011) and the Numeric pain rating scale was taken as it is a 11 point pain intensity numerical rating scale (PI-NRS) where 0 is no pain and 10 is worst possible pain. The NPRS takes less than 1minute to complete and is easy to administer and score (Pal and Velkumary 2004). 


\section{Journal of Exercise Science \& Physiotherapy Vol. 15 No. 1 (January to June) 2019 \\ ISSN: 0973-2020 (Print) $\quad \mathrm{I}_{2} \mathrm{OR}$ Impact Factor $=6.502 \quad$ ISSN: 2454-6089 (Online)}

\section{Results}

The raw data of the present study was analyzed in terms of MMDQ and NPRS for pain during the first day of menstruation. The pre and post differences were compared between Group A (Buteyko breathing) and Group B (Coherent breathing) using the t-test. SPSS (version 23) was used in statistical analysis. The pre and post scores of NPRS and MMDQ were recorded and the parametric dependent t-test was applied. Comparison of pre and post measurement was done by using t-test. Clinically both Group A (Buteyko breathing exercise) and Group B (Coherent breathing exercise) showed significant 5\% reduction in pain intensity, but Coherent breathing exercise showed better statistical results than Buteyko breathing exercise.

\section{Discussion}

The current study was intended in comparing the effectiveness of two types of breathing exercises namely Buteyko breathing and coherent breathing on pain. It is a known fact that in our body the sympathetic and parasympathetic systems work simultaneously, so the activation of one leads to the suppression of the other. In a study done by Choudhury et. al., (2011) it was shown that during the luteal and follicular phase of the menstrual cycle the parasympathetic activity is decreased thereby increasing the activity of the sympathetic system and hence causing increased pain perception (Choudhury et.al.,2011).Also it is known that there is increased synthesis of prostaglandins under the influence of the hormone progesterone, this synthesis of progestrone reaches its peak during shedding of myometrium. Prostaglandins being a vasoconstrictor causes ischemia of the myometrium causing increased myometrial contraction which causes pain perception during menstruation (Markum 1976). A study conducted by Pal and Velkumary (2004) on the effect of short term practice of breathing exercise on autonomic functions in normal human volunteers and they found that breathing exercise have been proved to increase the activity of the parasympathetic system and their study concluded that slow breathing when compared to fast breathing improves autonomic functioning. Previously a similar study on breathing exercise reported that there was increase in the production of IgG and beta endorphins which leads to decrease in pain perception (Proctor and Farquhar 2006). Buteyko breathing exercise is one such technique which consists of three components namely nasal breathing, control pause and relaxation. It is less time consuming, easy to administer. In the current study Group A was given Buteyko exercise which showed significant reduction in pain perception. The combined effect of nasal breathing, control pause and relaxation of buteyko exercise may be a possible reason for significant pain reduction in Group A participants Coherent breathing is a type of deep breathing exercise and deep breathing has shown to have a significant result in pain perception. A study done by Busch et. al., (2012) on the effect of deep and slow breathing on pain perception, autonomic activity and mood processing showed that the way of Breathing positively influences autonomic and pain processing, thereby identifying deep slow breathing along with relaxation as the essential feature in the modulation of sympathetic arousal and pain perception. Based on the results of above study, the present study may explain the possible reason of reduction in pain in Group B participants who received Coherent breathing exercise. In the present study, NPRS and MMDQ were used as outcome measures. NPRS is a valid and reliable scale to measure pain intensity, it is better as compared to Pain VAS in a way that it can be administered both verbally and in writing. High testretest reliability has been observed in both literate and illiterate patients ( $\mathrm{r}=0.96$ and 0.95 , respectively). Hence, this suggests that it may be a useful method for assessing menstrual distress. In the present study both the interventions showed significant pain reduction but Coherent breathing exercise had better results in terms of NPRS and MMDQ score than Buteyko breathing exercise. NPRS quantifies pain whereas MMDQ quantifies mood and autonomic functioning. Hence a reason for better results obtained in Group B participants when compared to Group A may be because 


\section{Journal of Exercise Science \& Physiotherapy Vol. 15 No. 1 (January to June) 2019 \\ ISSN: 0973-2020 (Print) I I OR Impact Factor =6.502 ISSN: 2454-6089 (Online)}

coherent breathing exercise being a deep breathing exercise it focuses on pain, mood processing and autonomic functioning while Buteyko exercise is controlled nasal breathing exercise and all the three components focuses only on pain.

\section{Conclusion}

The results obtained in the present study showed that both Buteyko breathing exercise and Coherent breathing exercise were effective significantly but Coherent breathing exercise was more significant statistically compared to Buyteko breathing exercise and hence it can be concluded that Coherent breathing exercise is more effective in subjects with primary dysmenorrhoea.

\section{References}

Busch V, Magerl W, Kern U, Haas J, Hajak G, Eichhammer P.2012. The effect of deep and slow breathing on pain perception, autonomic activity, and mood processing--an experimental study. Pain Med. Feb;13(2):215-28. doi: 10.1111/j.1526-4637.2011.01243.x. Epub 2011 Sep 21.

Chen C., Cho S.I., Damokosh A.I., Chen D., Li G., Wang X., Xu X.2000.Prospective study of exposure to environmental tobacco smoke and dysmenorrhea. Environmental Health Perspectives.Nov;108(11):1019.

Choudhury R., Jahan N., Sultana N., Akter R., Khanum A.A.2011. Parasympathetic nerve function status duing different phases of menstrual cycle in healthy young women. Journal of Bangladesh Society of Physiologist.6(2):100-7.

Dutta D.C. 2008.Textbook of Gynaecology including of contraception. $7^{\text {th }}$ edition. The Health Sciences Publisher, New Delhi.

Grandi G., Ferrari S., Xholli A., Cannoletta M., Palma F., Romani C., Volpe A., Cagnacci A.2012. Prevalence of menstrual pain in young women: what is dysmenorrhea?. Journal of Pain Research 5:169.

Iacovides S, Avidon I, Bentley A, Baker F.C. 2014b. Reduced quality of life when experiencing menstrual pain in women with primary dysmenorrhea. Acta Obstet Gynecol Scand 93:213-217.

Kaur A., Ray G., Mitra M. 2017.Comparing the Effectiveness of Connective Tissue Mobilisation and Kinesiotaping on Females with Primary Dysmenorrhea. Website: www. ijpot. com. Jul;11(3):70.

Markum R.A.1976. Assessment of the reliability of and the effect of neutral instruction on the symptom ratings on the Moos Menstrual Distress Questionnaire. Psychosomatic Medicine.38(3):163-172.

Pal G.K. and Velkumary S.2004. Effect of short term practice of breathing exercise on autonomic functions in normal human volunteers. Indian Journal of Medical Research.Aug 1;120(2):115.

Proctor M. and Farquhar C. 2006.Diagnosis and management of dysmenorrhoea. BMJ. 11;332(7550):11341138.

Reyes del Paso GA, Munoz Ladron de Guvera C, Montoro Cl. Breath holding during exhalation as a simple manipulation. Pain Medicine. 2015 Sep. 1;16(9):1835-41.

Sundell G., Milsom I., Andersch b.1990.Factors influencing the prevalence and severity of dysmenorrhoea in young women. BJOG: An International Journal of Obstetrics \& Gynaecology. 1990 Jul;97(7):58894. 